\title{
Photodynamic therapy for esophageal cancer
}

\author{
R LAMBERT, MD
}

\begin{abstract}
In photodynamic therapy, a photosensitizing drug is injected and then activated by light in the red spectral region to produce reactive, highly toxic singlet oxygen, which causes cell tissue and damage. When the distinction between tumoral and normal tissue is difficult at endoscopy, photodynamic therapy w preferable to any other conservative method of destruction. The main indications comprise genital, head and neck, bronchial, bladder and gastrointestinal tumours, particularly esophageal cancer (including squamous cell cancer and glandular neoplasia). Esophageal tumours should be class T1 or T2, at most, and detected at endoscopy. Dysplasia which is flat, sessile and multicentric may also be an indication for photodynamic therapy. Advanced esophageal cancers are contraindicated for photodynamic therapy. Can J Gastroenterol 1990;4(9): $612-615$
\end{abstract}

Key Words: Endoscopy, Esophageal cancer, Laser, Photodynamic therapy

\section{La thérapie photodynamique du cancer de l'oesophage}

RESUME: La thérapie photodynamique (TPD) est une forme particulière de photochimiothérapie consistant à injecter une substance photosensibilisante et à l'activer dans la partie infrarouge du spectre de la lumière. Le photosensibiliseur activé porte les molécules d'oxygène dans leur état singulet excité (oxygène singulet), un état hautement toxique provoquant des lésions cellulaires et tissulaires. Quand la distinction entre tissus tumoral et normal est difficile à déterminer sous endoscopie, la thérapie photodynamique est préférable à tout autre méthode conservatrice de destruction. Elle est principalement indiquée dans les tumeurs des organes génitaux, de la tête et du cou, des bronches, de la vessie et des voies gastro-intestinales - le cancer de l'oesophage surtout (épithélioma malpighien et néoplasie glandulaire). Les tumeurs de l'oesophage devraient être de catégories T1 ou T2, au plus, et détectées sous endoscopie. La TPD peut également servir au traitement de la dysplasie se révélant par des nodules aplatis, sessiles et multicentriques mais elle est contre-indiquée pour les cancers avancés de l'oesophage.

Gastroenterology Unit and INSERM U 45, Hospital E, Herrisot, France

Correspondence and reprints: Dr R Lambert, Gastroenterology Unit and INSERM U 45 ,

Hospital E, Herriot 69437 Lyon, Cedex 03-France
P HOTODYNAMIC THERAPY IS A FORM of photochemotherapy in whicha photosensitizing drug is injected and then activated by an appropriate light: wavelength, usually in the red spectral region. The activated photosensitizer converts oxygen molecules in its sur: rounding into reactive, highly toxic singlet oxygen, which causes cell and tissue damage. The photosensitizer used in clinical applications of photo. dynamic therapy is an hematoporphyrin derivative; the light sourceis a laser. The prolonged tumour retention of the hematoporphyrin derivative compared to normal tissues has led to applications for tumours $(1,2)$.

Photodynamic therapy has a solid experimental basis and excellent un. derlying principles, but adequate clinical trials to assess its practical value have been slow to develop. Many fac. tors account for this discrepancy. Until recently, it was difficult to get a regular supply of hematoporphyrin derivative. Medical laser sources are sophisticated, costly, and require close technical sur. veillance. More importantly, the initial uses - palliation of nonsuperficial cancer - were not shown to be favorable compared with curative attempts on superficial lesions. Finally, photodynamic 
therapy as a potential alternative therapy to surgery for poor surgical risk patients has been poorly communicated to clinicians so that recruitment for trials has been difficult.

In clinical oncology, the indication for photodynamic therapy is based upon principles typical in any type of tumour therapy.

The tumour cells should have good susceptibility to this procedure of destruction. This depends upon the histology and location of the tumour (geometry of irradiation).

The tumour should be superficial (classification T1). Such lesions are usually asymptomatic, and careful endoscopic screening of high risk patients is necessary to promote indications.

There should be no alternative or easy procedure of destruction (such as a local tumorectomy). When the distinction between tumoral and normal tissue is difficult at endoscopy, photodynamic therapy is preferable to any other conservative method of destruction.

The main indications comprise genital, head and neck, bronchial and bladuer tumours, as well as tumours of the digestive tract. Esophageal cancer is the most appropriate indication among digestive tract tumours.

\section{RATIONALE IN ESOPHAGEAL CANCER}

Tumour type: Squamous cell cancer is the usual indication. Potential extension to severe squamous cell dysplasia is possible when the lesions are not accessible to snare resection (ie, if lesions are flat and multicentric).

Glandular neoplasia in the esophagus is less frequent. It develops in columnar mucosa, usually in relation to reflux. Neoplasia in Barrett's epithelium may be located either in the mid (circumferential columnar lining) or lower esophagus (noncircumferential lining). In the latter condition any distinction between neoplasia at the cardia and neoplasia in the esophagus is artificial. Esophageal glandular neoplasia may be classified as adenomatous dysplasia or adenocarcinoma.

Endoscopic morphology of the tumour: At the superficial stage, esophageal neoplasia does not interfere with peristalsis and is not obstructive. Slight alterations in mucosal architecture results in depression, elevation or a mixed pattern. In squamous neoplasia the mucosal pattern may appear normal without coloration. After spraying with Lugol's iodine solution, the neoplastic area remains unstained in contrast to the dark brown of the normal mucosa. In glandular neoplasia the endoscopic pattern of superficial cancer or dysplasia is characterized by longitudinal nonulcerated sessile elevations (adenomatous type) of the mucosa which are often circumferential.

As for the geometry of irradiation, the tubular shape of the esophagus allows easy and effective irradiation without any blind areas. Evaluation of the surface of the lesion is required $f$ or dosimetry (duration of irradiation); for this purpose the height of the lesion is the main parameter. The circumferential light repartitor is preferred for irradiation in circumferential and noncircumferential lesions. In the latter condition, irradiation is therefore distributed in part to the normal mucosa; dosimetry should take this into account.

Staging of the tumour: Following surgical treatment the tumour is staged as superficial (limited to mucosa and submucosa, T1) or nonsuperficial (muscular or periesophageal extension, T2-T4) in the operative specimen, according to the 1987 TNM classification. Superficial cancer of the squamous mucosa may be further classified as intraepithelial (muscularis mucosae not invaded), intramucosal and submucosal. In situ malignancy (no lymphatic invasion) characterizes intraepithelial cancer; this is the only type without potential for lymphatic extension. In glandular neoplasia of the columnar-lined epithelium, the lesion is classified into two groups only: intramucosal or submucosal. The potential for lymphatic extension is less in the intramucosal type versus submucosal, but not nil.

In the absence of surgery, staging is done via endoscopy, computed tomography or echoendoscopy. The latter procedure characterizes the tumour superficially, with an over $90 \%$ efficacy when the second hyperechoic layer is not interrupted. The superficial pattern of a tumour at endoscopy is confirmed at echoendoscopy in no more than $60 \%$ of cases. Furthermore, echoendoscopy does not contribute to a distinction between intraepithelial and other types of superficial squamous neoplasias. Therefore, a local tumorectomy procedure such as photodynamic therapy should be used in conjunction with systemic (chemotherapy) or regional (radiotherapy) agents in a curative protocol.

The diagnosis of squamous or glandular dysplasia in the esophagus is based on biopsy at endoscopy. Difficulties in confirming this diagnosis in the squamous epithelium should be kept in mind. Cellular alterations in reflux esophagitis should not be mistaken for premalignant change. The superficial cellular alterations connected with viral infection in condyloma of the esophagus are not linked to neoplasia, while dysplasia originating from the deep epithelial layer is a premalignant lesion. Dysplasia is of course always staged as superficial at echoendoscopy. Therefore, exploration is recommended as a further control of the biopsies.

Decision analysis: In esophageal squamous cell cancer the overall prognosis is very poor and treatment usually does not achieve a cure. The selection of a nonsurgical procedure versus esophagectomy depends upon patient age, tumour morphology and associated cancerous or noncancerous diseases. When a nonsurgical protocol is adopted, its objective is either partial destruction of the tumour (palliation of symptoms), extensive destruction (palliation and prolonged evolution through a radical protocol), or total destruction (curative treatment).

Palliative protocols are based on widening of the esophageal lumen using a thermal neodymium YAG (Nd:YAG) laser in monotherapy. Radical protocols in superficial and nonsuperficial cancer combine Nd:YAG laser, chemotherapy (cisplatinum and 5-fluorouracil), and radiotherapy. Photodynamic therapy will be used instead of Nd:YAG in a limited group of patients. 
It should be used when a cancer detected at endoscopy has a superficial pattern and is confirmed as superficial at echoendoscopy ( $\mathrm{T} 1$ group). If the patient is in poor health, old or has associated disease, irradiation can be performed as monotherapy. This concerns also patients previously treated by radiotherapy for esophageal cancer, with recurrence of the superficial type. If the patient is young or in good health, the protocol is proposed as a curative alternative to surgery. Therefore, photodynamic therapy should always be completed by radiotherapy (60 Gy equivalent) two months later.

When the superficial pattern of the tumour at endoscopy is not confirmed at echoendoscopy (T2 classification), photodynamic therapy may still be used if there is limited invasion of the muscular layer. However, irradiation is performed after a chemotherapy session. The multimodal protocol combines chemotherapy, photodynamic therapy and radiotherapy.

When muscular invasion is classified as extensive at echoendoscopy, the Nd:YAG laser multimodal protocol is preferred

In esophageal adenocarcinoma that has developed in columnar epithelium, surgical treatment (esophagectomy) is always preferred in superficial and nonsuperficial cancer, as it is potentially curative. A nonsurgical protocol is adopted only if there exist contraindications to surgery (poor health status or old age). Then Nd:YAG laser and/or stenting are adapted to the treatment of advanced cancer. Photodynamic therapy should be proposed if a superficial pattern at endoscopy is confirmed at echoendoscopy; this concerns very few patients.

In dysplasia, indications for photodynamic therapy should be developed for the treatment of flat, sessile and multicentric lesions. Treatment of these depends on the promotion of careful endoscopic screening of the esophageal mucosa. In severe dysplasia the choice is between radical surgery for a premalignant lesion and local destruction by photodynamic therapy. Dysplasia of the squamous epithelium in association with cancer should be treated simultaneously with the main tumour. Severe dysplasia occurring in a flat condyloma will be treated by this procedure. Dysplasia of columnar-lined epithelium is more common and may be detected during surveillance of a Barrett's esophagus. The response of columnar dysplastic mucosa to photodynamic therapy is quite specific. After destruction, healing should be placed under the control of an antisecretory agent (a proton pump inhibitor) and/or surgical correction of the reflux. The regenerated mucosa may revert to squamous in the treated area.

\section{METHOD OF PHOTODYNAMIC THERAPY}

The photosensitizing agent hematoporphyrin derivative, or its dimeric ether, DHE, is injected intravenously in the course of a DW $5 \%$ perfusion. The dosage most often used is 2.0 to 2.5 $\mathrm{mg} / \mathrm{kg}$ body weight. Endoscopic irradiation is performed using a monochromatic $(630 \mathrm{~nm})$ laser beam of red light transmitted through a flexible quartz fibre. The argon pumped-dye laser is still the standard equipment. The delivered light dose should be in the range of 100 to $200 \mathrm{~J} / \mathrm{cm}^{2}$, with a power density in the range of 100 to 500 $\mathrm{mW} / \mathrm{cm}^{2}$. Tumour necrosis begins within $24 \mathrm{~h}$; its duration varies according to tumour depth (one week to one month). Stricture may result from the treatment of circumferential lesions at the healing stage. The major toxicity of photodynamic therapy is skin photosensitivity, which can be prevented by protection of the patient from direct sunlight during the first month. Other side effects reported include nausea, vomiting and liver toxicity. Usually these are very mild.

During the period of August 1983 to January 1990, the authors treated 100 patients with esophageal neoplasia via a nonsurgical protocol including photodynamic therapy. This included 14 nonsuperficial and 86 superficial cancers (determined by endoscopy) or severe dysplasia. After 1987, superficial character was determined by endosonography: a tumour with a superficial endoscopic pattern was confirmed as
T1 (muscular layer not invaded) in 609 of cases or T2 (muscular layer invaded) in $40 \%$. The histology of the esophageal neoplasias was as follows: adenocar cinoma in 17 cases (six nonsuperficial cancers, eight superficial cancers, three with dysplasia); squamous neoplasia in 83 cases (seven nonsuperficial cancers, 76 superficial cancers or severe dysplasia).

The patients were injected intra. venously with, according to the period Photofrin I, Photofrin II (QLT, Vancouver), or hematoporphyrin derivative from Quentron (Australia). The dose varied from 2.0 to $3.0 \mathrm{mg} / \mathrm{kg}$ body weight. Irradiation $72 \mathrm{~h}$ later was per. formed with an SP dye laser pumped by an argon laser through a quartz fibre with an annular light repartitor at its tip. Power range varied from $750 \mathrm{w}$ $1250 \mathrm{~mW}$, power density from 100 to $500 \mathrm{~mW} / \mathrm{cm}^{2}$. For one full month after treatment, the patients avoided diret exposure to sunlight. Photodynamic therapy was proposed as monotherapy in half of the patients. The other half was included in a multimodal protocol receiving chemotherapy (cisplatinum, 5-fluorouracil and/or radiotherapy) (3). Radiation began after an interval of two months following photodynamic therapy, to prevent cutaneous complications of photosensitization.

\section{RESULTS OF PHOTODYNAMIC THERAPY}

It is not clear whether photo. dynamic therapy in nonsuperficial esophageal cancer offers any advantage over Nd:YAG laser therapy. Indeed photodynamic therapy in such cases is often associated with prolonged tumour necrosis resulting in impaired relief of dysphagia; there is a risk of complica. tions such as perforation, hemorrhage or inflammatory stricture. Furthermore, prompt regrowth of the tumour is the rule. McCaughan (4) treated 40 patients (adenocarcinoma and squamous cell cancer). Jin (5) treated 20 patients with squamous cell cancer and 71 with advanced cancer of the cardia. Thomas (6) treated 15 patients with squamous cell cancer. Due to poor results observed in the first cases, ad. vanced squamous cell cancer and 
adenocarcinoma were considered as contraindications to photodynamic therapy.

Results obtained in superficial squamous cell cancer are much better. Tian (7) treated 13 patients with good results in 12 . Four complete remissions in six treated patients were reported by Hayata (8); five of six were reported by Tajiri (8) and six of eight by Monnier (10).

In the author's series (100 patients treated) good results were obtained only in superficial neoplasia determined by endoscopy. Prompt recurrence occurred in all other cases. The rate of complete tumour destruction assessed by a negative biopsy at endoscopy three months after treatment reached $75 \%$ in superficial cancers confirmed at echoendoscopy (T1) and only 50\% in false superficial cancers (T2). The rate of complete destruction was higher in superficial squamous cell neoplasia than in adenocarcinoma. The average period of follow-up is 22 months in living patients. In the others, the cause of death was unrelated to the evolution of esophageal cancer in $60 \%$. Indeed, in pacients with superficial squamous neoplasia the rate of death from other diseases (associated ear, nose and throat cancer, bronchial cancer, liver cirrhosis and alcoholic cardiomyopathy) was very high. The actuarial survival rate in squamous cell neoplasia at two years

\section{REFERENCES}

1. Bown SG. Photodynamic therapy. Basic principles. In: Riemann JF, Ell C, eds. Lasers in Gastroenterology. New York: Thieme Medical Publishers, 1989:85-132.

2. Dougherty TJ. Photodynamic therapy. Adv Exp Med Biol 1985; 193:313-28.

3. Lambert R, Sabben G, Souquet JC, et al. Photodynamic therapy. Clinical experience. In: Riemann JF, Ell C, eds. Lasers in Gastroenterology. New York: Thieme Medical Publishers, 1989:93-9. 4. McCaughan J, Nims TA, Guy JT, et al. reached $55 \%$ when all causes of death were considered. It increased to $78 \%$ if death related to the evolution of esophageal cancer was the only factor considered. The respective figures for cases that were confirmed as superficial at echoendoscopy (T1) were 65 and $100 \%$. For false superficial cancer (T2) they were lower: 43 and $75 \%$.

\section{CONCLUSIONS}

Photodynamic therapy in esophageal cancer should be reserved for superficial neoplasias detected at endoscopy (nonstenosing tumour with esophageal peristalsis maintained). For large tumours deeply penetrating the esophageal wall, photodynamic therapy is contraindicated; this is determined by endoscopic study. Better results are obtained in squamous cell versus glandular neoplasia and when the tumour is staged T1 at echoendoscopy versus when the muscular layer is invaded (T2). Photodynamic therapy in squamous cell neoplasia should be included in a multimodal protocol (chemotherapy and/or radiotherapy) when the patient's condition is good.

Indications in adenocarcinoma can be summarized as follows: when the cancer is staged as superficial at echoendoscopy (T1), photodynamic therapy will be proposed only in patients at high risk for surgery. Low risk patients should preferably be operated on. Tumours

Photodynamic therapy for esophageal tumours. Arch Surg 1989;124:74-80.

5. Jin ML, Yang BQ, Zhang W, et al. Photodynamic therapy for the treatment of advanced gastrointestinal tumours. Lasers Med Sci 1989;4:183-6.

6. Thomas RIS, Morstyn G. Photodynamic therapy for esophageal cancer Lasers Med Sci 1988;3:A33.

7. Tian ME, Qui SL, Ji Q. Preliminary results of hematoporphyrin derivative laser treatment for 13 cases of esophageal carcinoma. Adv Exp Med staged T2 are operated or treated by Nd:YAG thermal laser in a palliative protocol. The superficial lesion clas. sified as severe dysplasia via biopsy is an elective indication for photodynamic therapy. Cases with moderate dysplasia should be included in a surveillance program and not treated at this stage.

Indications in squamous neoplasia suggest that photodynamic therapy is an alternative to surgery (esophagectomy) in patients with superficial cancer such as those detected at endoscopy. The alternative is accepted in 'low risk' and young patients when the tumour is staged T1 at echoendoscopy. Photodynamic therapy is then included in a multimodal protocol and the patients receive a course of radiation (without chemotherapy) two months after the laser treatment. When the tumour is staged T2 at echoendoscopy, photodynamic therapy is preferred to surgery only in poor risk patients. Chemotherapy is included from the beginning of the protocol and radiotherapy performed 21 months later. In severe squamous cell dysplasia associated with reflux esophagitis or papilloma virus infection (condyloma), photodynamic therapy is an elective indication with lesions which are flat and multicentric. Patients with moderate dysplasia should not be treated and should be included in a surveillance program.

Biol 1985;193:21-5.

8. Hayata Y, Kato H, Okitsu H, et al. Photodynamic therapy with hematoporphyrin in cancer of the upper gastrointestinal tract. Semin Surg Oncol 1985;1:1-11.

9. Tajiri H, Daikuzono N, Joffe S, et al. Photoradiation therapy in early gastrointestinal cancer. Gastrointest Endosc 1987;33:88-90.

10. Monnier P, Fontolliet C. PDT of early pharyngeal, oesophageal and bronchial carcinoma. Lasers Med Sci 1988;3:A31. 


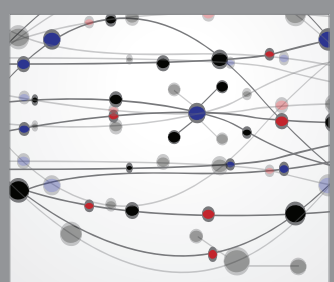

The Scientific World Journal
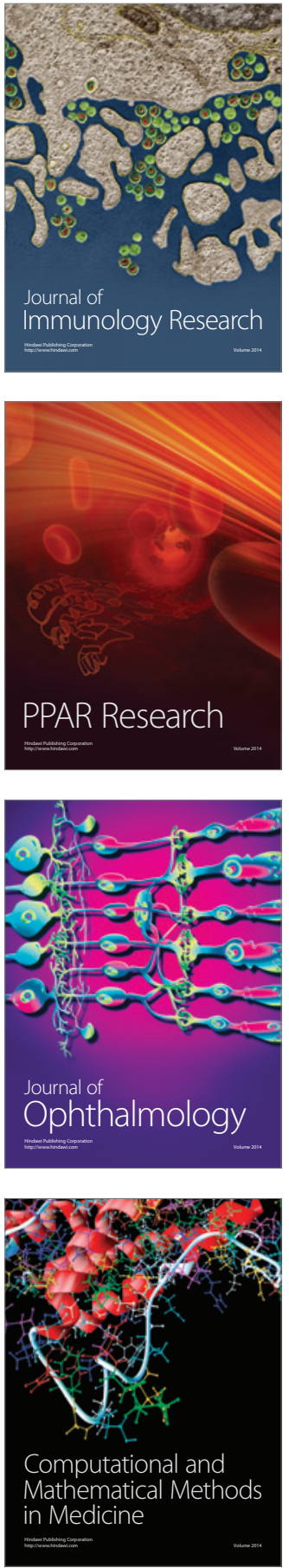

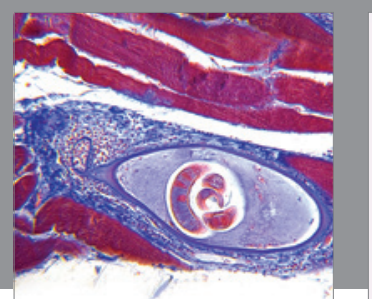

Gastroenterology Research and Practice

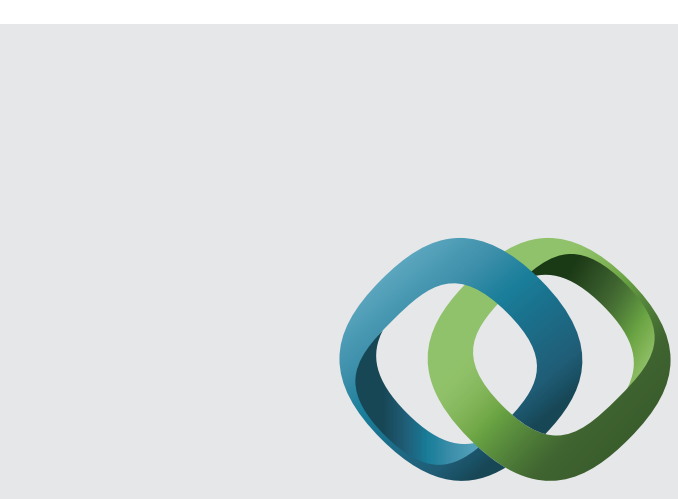

\section{Hindawi}

Submit your manuscripts at

http://www.hindawi.com
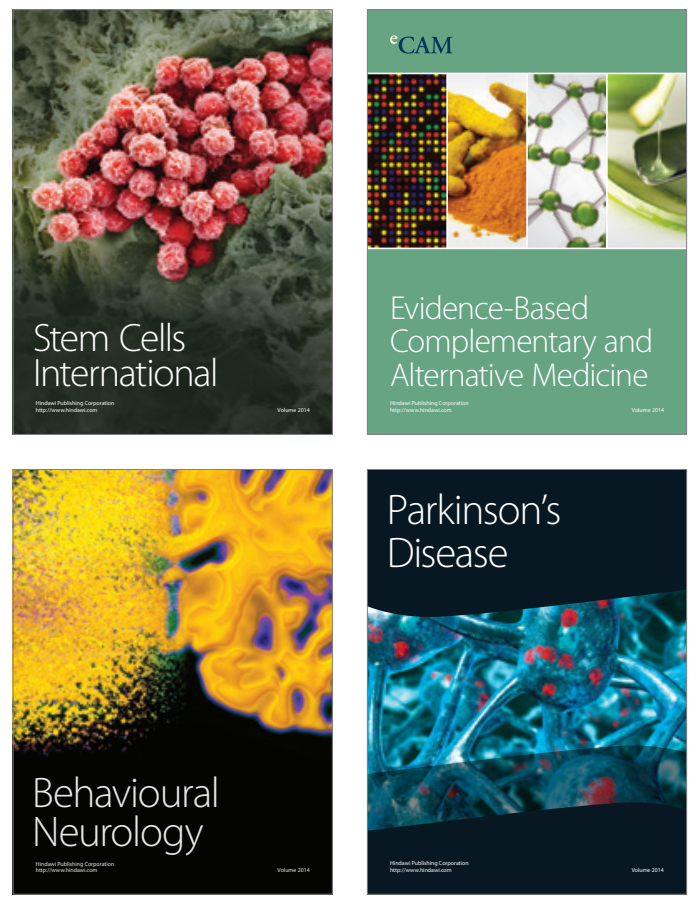
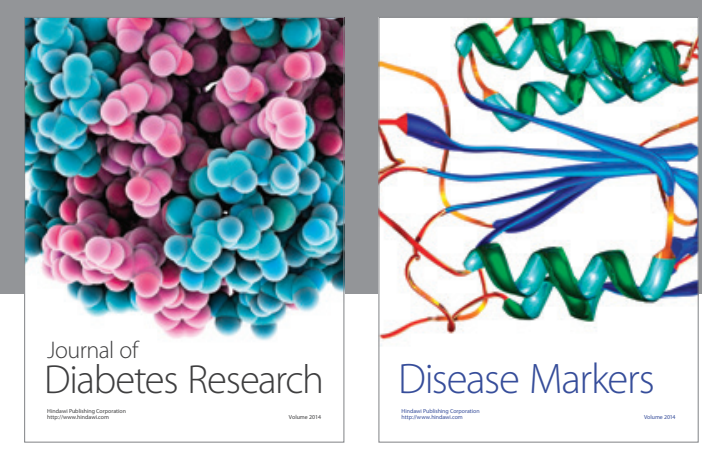

Disease Markers
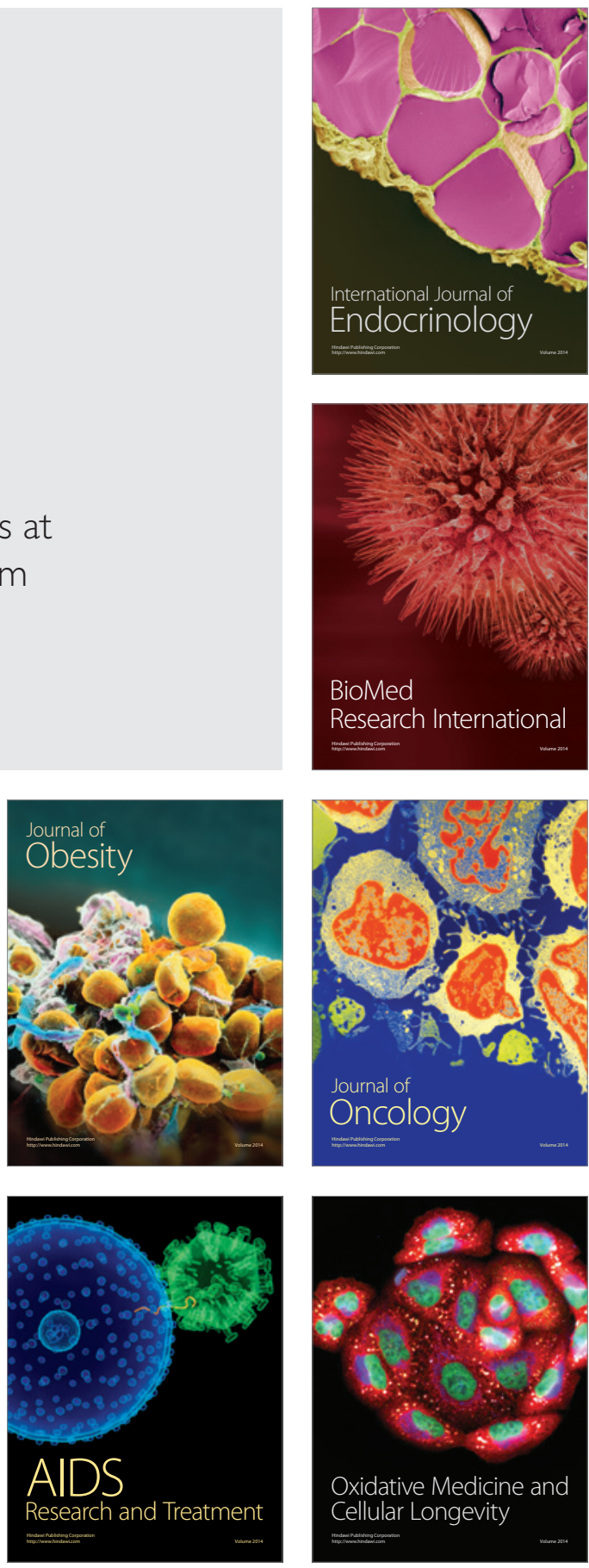